\title{
Tubular aggregates: their association with myalgia
}

\author{
ENAYAT NIAKAN, ${ }^{*}$ YADOLLAH HARATI,${ }^{*}$ MORIS J DANON $\dagger$ \\ From the Department of Neurology, Baylor College of Medicine, Houston* and Department of Neurology $\dagger$ \\ University of Illinois Medical Center, Chicago, USA
}

SUMMARY Three thousand consecutive muscle biopsies were reviewed for the presence of tubular aggregates and their association with clinical symptomatology. Tubular aggregates were detected in 19 patients $(0.6 \%)$. Twelve of these nineteen patients had severe myalgia, and the most abundant tubular aggregates were found in biopsies of patients with myalgia. Seven patients had only myalgia as their clinical symptomatology with normal physical examination. An additional five patients with tubular aggregates and myalgia had concomitant amyotrophic lateral sclerosis (2) or neuropathy (3). The high incidence of myalgia associated with tubular aggregates in our patients and the fact that tubular aggregates originate from sarcoplasmic reticulum suggest a role played by this structure in the pathogenesis of myalgia.

Tubular aggregates are visible by light microscopy within Type II muscle fibres as a mass in the subsarcolemmal region with strong NADH-tetrazolium reductase (NADH-TR) and negative succinic dehydrogenase reactions. Ultrastructurally they are closely packed, parallel, double-walled tubules which appear to be a proliferation of sarcoplasmic reticulum.' The diagnostic importance of tubular aggregates is uncertain because they are present in muscles of patients with a wide variety of disorders. $^{1-21}$ Recently tubular aggregates have been described in muscle biopsy specimens of patients with myalgia who are otherwise normal. ${ }^{21-26}$ The fact that tubular aggregates originates from sarcoplasmic reticulum, which has an important role in regulation of muscle contracture and relaxation, suggests a relationship between tubular aggregates, sarcoplasmic reticulum and myalgia. To clarify this relationship, we reviewed muscle biopsy specimens for the presence of tubular aggregates and their association with clinical symptomatology.

\section{Methods}

We analysed all 3000 muscle biopsy specimens at our institution in the preceeding seven years. All were from the

Presented in part at the Thirty-Sixth Annual Meeting of the American Academy of Neurology, Boston, April, 1984

Address for reprint requests: Dr Harati, Department of Neurology, Baylor College of Medicine, 6501 Fannin, Houston, Texas 77030 , USA.

Received 5 June 1984 and in revised form 27 August 1984 Accepted 1 September 1984 quadriceps or biceps muscles. For histochemical studies, tissue had been frozen with liquid nitrogen, sectioned at $10 \mu$ in a cryostat, and stained with the following methods: Modified Gomori Trichrome (TRI), NADH-TRT adenosine triphosphatase (ATPase) at $\mathrm{pH} 9.4$, succinie dehydrogenase and phosphorylase. For electon microse is copy, tissue had been fixed in $1 \%$ glutaraldehyde buffere $\mathbb{D}$ at $\mathrm{pH} 7.4$ for $1 \frac{1 / 2}{\mathrm{~h}}$ and then fixed in $1 \%$ osmium tetroxides $\overrightarrow{\mathbb{D}}$ for another hour. Some material was then embedded iff Epon and sections $1 \mu$ thick were cut for all blocks, staines with toluidine blue and examined under the light microso을 copy. Thin sections for electron microscopy were ther ${ }^{+}$ stained with uranyl acetate and with lead citrate.

\section{Results}

Among 3000 patients $19(0 \cdot 6 \%)$ had histochemically typical tubular aggregates; 17 patients were male and two female. With the TRI stain, the tubular aggregates appeared as bright red staining subsarcolemmal material. Tubular aggregates were seen only in the Type II fibres, were darkly stained by the NADH-TR, and unstained by ATPase and succinate dehydrogenase (fig 1). Further studies including electron microscope and epoxy resin histology were done in some cases which showed closely packed, parallel, double-walled tubules (fig 2).

Five biopsies showed less than $1 \%$ fibres affected with tubular aggregates, six between $1-10 \%$, and eight had more than $10 \%$ fibres with tubular aggregates. This was determined after obtaining a $100 \times$ photograph of each biopsy specimen and counting the number of tubular aggregates per 200 muscle fibres.

Clinically, 12 of 19 patients had severe myalgia, 

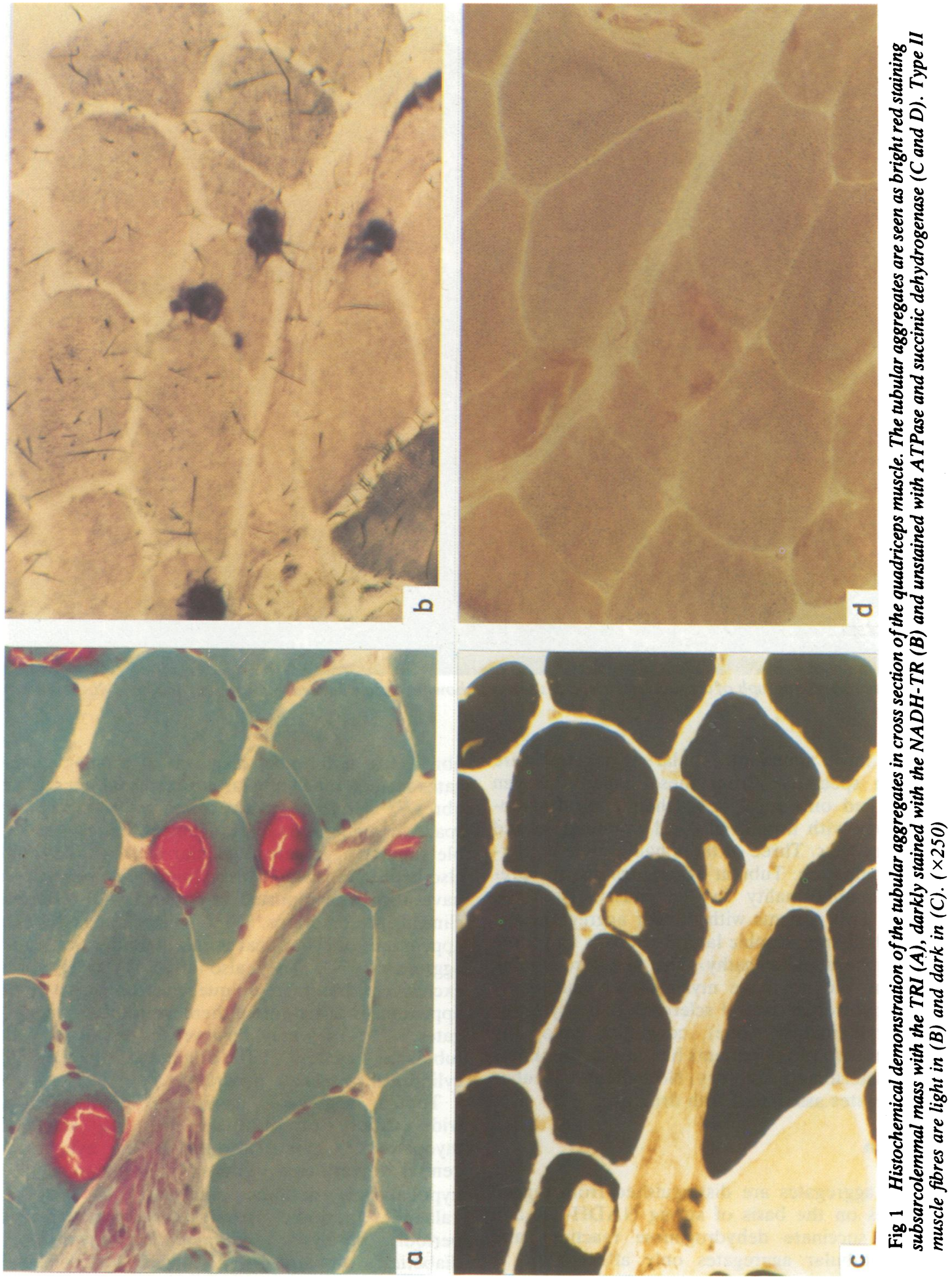


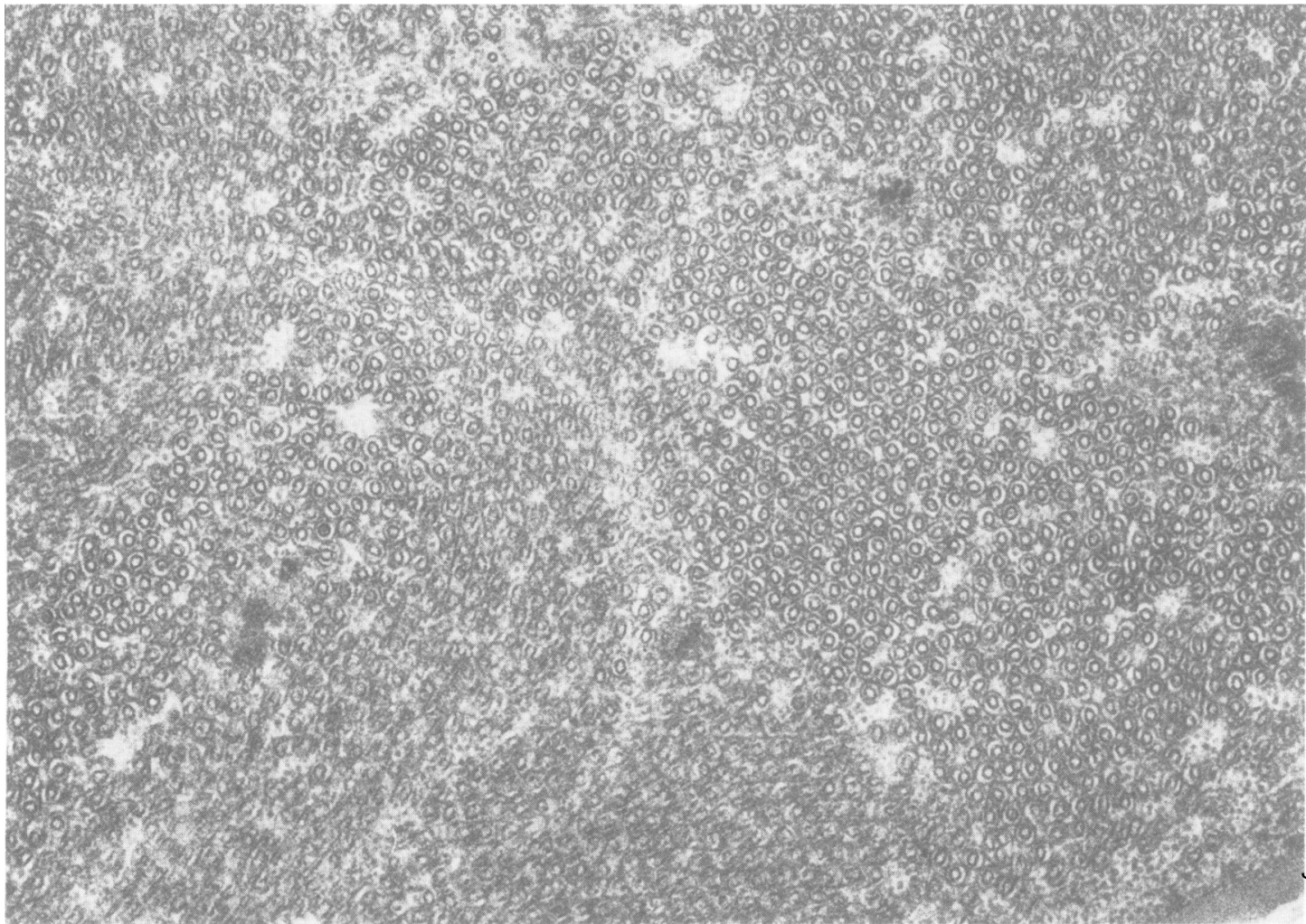

Fig 2 Electron micrograph of the same muscle, cross section, showing closely packed, parallel and double-walled tubules. $(\times 50,000)$

and the most abundant tubular aggregates were found in specimens from these patients. Seven patients had only myalgia as their clinical symptomatology with normal physical examination and laboratory tests. Three of them had muscle tenderness on palpation. Tubular aggregates was the predominant abnormality in the muscle of these patients. Two patients with tubular aggregates and myalgia had amyotrophic lateral sclerosis and three had peripheral neuropathy. The seven remaining cases who did not have myalgia had neuropathy (two) amyotrophic lateral sclerosis (one), systemic lupus erythematosis (one), congenital myopathy (one), and two of the patients had muscle weakness with undetermined aetiology. No patient had tubular aggregates associated with drug use.

\section{Discussion}

Tubular aggregates are distinguished from ragged red fibres on the basis of strong NADH-TR and negative succinate dehydrogenase reactions and because tubular aggregates only affect Type II fibres. The differential diagnosis of tubular aggregates include other tubular structures within muscle fibres. ${ }^{12}{ }^{16}$ Honeycomb structures are round empty spaces in an hexagonal arrangement that can resemble tubular aggregates. Tubular aggregates should also be differentiated from cylindrical spirals, which have also been reported in patients with different clinical presentations. ${ }^{1127-29}$ Histochemically, the appearance of cylindrical spirals is similar to tubular aggregates."12729 They also affect Type II fibres exclusively, but their unique electron microscopic appearances differentiate them from tubular aggregates. ${ }^{1127-29}$ They may, however, be associated with tubular aggregates. ${ }^{11}{ }^{27}$ The origin and significance of cylindrical spirals are uncertain.

Tubular aggregates have been reported in a wide variety of disorders including alcoholic myopathy, ${ }^{16}{ }^{17}$ gyrate atrophy of the eye, ${ }^{2}$ congenital myasthenia gravis, ${ }^{4-614}$ myotonia, ${ }^{7521}$ hypokalaemic periodic paralysis, ${ }^{18418-21}$ hyperkalaemic periodic paralysis,' normokalaemic periodic paralysis, ${ }^{13}$ inflammatory myopathy, ${ }^{2}$ diabetic amyotrophy, ${ }^{3}$ hyperaldosteronism, ${ }^{12}$ 
prophyria cutanea tarda and chronic drug exposure.' A similar abnormality has been found in muscles of mice after anoxia ${ }^{30}$ and after local injection of botulinum, ${ }^{31}$ tetanus toxin, ${ }^{31}$ and perhexiline maleate $^{33}$ and in murine dystrophic heterozygotes. ${ }^{34}$ Tubular aggregates have also been described in association with myalgia in otherwise normal individuals. $^{21-26}$ Twelve of 19 of our patients with tubular aggregates had severe myalgia. Seven patients had myalgia as their sole clinical symptomatology and despite extensive investigation, no definite diagnosis could be made. Like patients reported by Rosenberg et al, ${ }^{21}$ myalgia was not necessarily precipitated by exercise. Three of these cases had severe muscle tenderness on palpation. Tubular aggregates was the prominent abnormality in the muscle of these patients. Five other patients with tubular aggregates and myalgia had concomitant amyotrophic lateral sclerosis or neuropathy. In these patients myalgia was one of their major complaints.

It is generally accepted that tubular aggregates originate in the sarcoplasmic reticulum, but their pathogenesis is unknown. Based on the finding of tubular aggregates in muscle biopsies of patients chronically using large amounts of analgesicnarcotic drugs and alcohol, ${ }^{16}$ it has been postulated that they may form in response to exogenous or endogenous toxin. However, these reports failed to indicate why these medications were taken or why the muscle biopsies were performed, ${ }^{116}$ and it may be that the reason for chronic analgesic use was for relief of myalgia. ${ }^{16}$ It has also been suggested that tubular aggregates may represent adaptive hyperplastic sarcoplasmic reticulum derivatives to improve calcium uptake in alcoholic myopathy. ${ }^{17}$ However, there was no history of chronic drug use or alcoholic abuse in our cases. We also doubt that this is a virus-induced lesion as postulated by Dunkle et $\mathrm{l}^{18}$ and Bergman, ${ }^{89}$ since no evidence of viral infection of muscle was seen. There is thus no satisfactory explanation for the formation of tubular aggregates, nor for their occurrence in a wide variety of diseases, but there may be an explanation of the high incidence of myalgia associated with them.

Sarcoplasmic reticulum has an important role in contraction and relaxation of muscle. The function of the sarcoplasmic reticulum is regulation of the calcium concentration in the vicinity of actin and myosin interaction. This regulation of calcium concentration is the control factor for turning on and off the basic contractile unit, the sarcomere. It is well known that malfunction of sarcoplasmic reticulum interferes with normal contraction and relaxation, and it may cause myalgia. The fact that tubular aggregates originates from sarcoplasmic reticulum and the high incidence of myalgia associated with tubular aggregates in our patients and previous reports of similar cases, ${ }^{21-26}$ suggest a role played by this structure in the pathogenesis of myalgia. The relationship between tubular aggregates, sarcoplasmic reticulum and calcium metabolism may also explain why tubular aggregates are seen only in the Type II fibres. Type II fibres have considerably more sarcoplasmic reticulum than the Type I fibres. ${ }^{35}$ In Type II fibres, the sarcoplasmic reticulum is possibly qualitatively different from that of the Type 1 fibre.' Also it has been shown in rabbits that white muscle fibres (Type II) have more active calciumconcentrating microsoms than red muscle fibres (Type 1). ${ }^{36}$ These structural differences may predispose Type II fibres to tubular aggregates formation.

In spite of relationships between tubular aggregates, sarcoplasmic reticulum and myalgia, tubular aggregates are not always associated with myalgia. There may be different types of tubular aggregates, ${ }^{1216173738}$ but there are classic forms of tubular aggregates in patients who never complain of myalgia. Also, in our patients who had electron microscopic studies, the pattern of tubular aggregates was similar in cases with or without myalgia. Degree of muscle fibre involvement with tubular aggregates may be an important factor for appearance of myalgia since the most abundant tubular aggregates were found in our patients with myalgia.

Based on our observation and review of literatures, we suggest the association of myalgia and tubular aggregates may be important. Although myalgia is the most prominent feature in the symptomatology of a wide variety of conditions, in many patients no firm diagnosis can be made despite extensive investigation. ${ }^{39}$ Therefore, the presence of tubular aggregates in patients complaining of maylgia may be a hallmark and should not be regarded as a nonspecific finding. Recognition of these combinations in time may lead to a better understanding of the significance of tubular aggregates. This relationship between tubular aggregates, sarcoplasmic reticulum and myalgia may also have important therapeutic implications, since drugs which are known to affect these structures may prove beneficial in alleviating symptoms of myalgia.

We thank Mr Karam Matta, and Ms C Zultner, for the technical assistance. Supported in part by a grant from the Frost Foundation.

\section{References}

${ }^{1}$ Engel WK, Bishop DW, Cunningham GG. Tubular aggregates in Type II muscle fibers: Ultrastructural and histochemical correlation. Ultrastructure Research 1970;31:507-25. 
${ }^{2}$ Sipila I, Simell O, Rapola J, et al. Gyrate atrophy of the choroid and retina with hyperornithinemia: Tubular aggregates and Type II fibre atrophy in muscle. Neurology (Minneap) 1979;29:996-1005.

${ }^{3}$ Chokroverty S, Reyes MG, Rubino FA, Tonaki H. The syndrome of diabetic amyotrophy. Ann Neurol 1977;2:181-4.

${ }^{4}$ Morgan-Hughes JA, Lecky BRF, London DN, Murray NMF. Alteration in the number and affinity of junctional acetylcholine receptors in a myopathy with tubular aggregates. Brain 1981;104:279-95.

${ }^{5}$ Engel AG, Lambert EH, Mulder DM, et al. A newly recognized congenital myasthenic syndrome attributed to a prolonged open time of the acetylcholineinduced ion channel. Ann Neurol 1982;11:553-69.

6 Dobkin BH, Verity MA. Familial neuromuscular disease with Type I fibre hypoplasia, tubular aggregates, cardiomyopathy, and myasthenic features. Neurology (Minneap) 1978;28:1135-40.

${ }^{7}$ Schotland D. Ultrastructural abnormalities in myotonic dystrophy including an unusual $\mathrm{T}$ system alteration. $J$ Neuropathol Exp Neurol 1968;27:109-10.

${ }^{8}$ Bergman RA, Afifi AK, Dunkle LM, Johns RJ. Muscle pathology in hypokalemic periodic paralysis with hyperthyroidism. Hopkins Med J 1970;126:88-94.

' Bergman RA, Afifi AK, Dunkle LM, Johns RJ. Muscle pathology in hypokalemic periodic paralysis with hyperthyroidism. Hopkins Med J 1970;126:101-18.

${ }^{10}$ Odor DL, Patel AN, Pearce LA. Familial hypokalemic periodic paralysis with permanent myopathy. J Neuropath Exp Neurol 1967;26:98-114.

"Carpenter S, Karpati G, Robitaille Y, Melmed C. Cylindrical spirals in human skeletal muscle. Muscle Nerve 1979; 2:282-7.

12 Gallas M. Myopathy with hyperaldosteronism. J Neurol Sci 1977; 32:337-45.

${ }^{13}$ Meyers KR, Gilden DH, Rinaldi CF, Hansen JL. Periodic muscle weakness, normokalemia and tubular aggregates. Neurology (Minneap) 1972;22:269-79.

14 Johns TR, Campa JF, Adelman LS. Familial myasthenia with "tubular aggregates" treated with Prednisone. Neurology (Minneap) 1973;23:426.

${ }^{15}$ Schiffer D, Giordana MT, Monga G, Mollo F. Histochemistry and electron microscopy of muscle fibres in a case of congenital paramyotonia. $J$ Neurol 1976;211: 125-33.

${ }^{16}$ Chui L, Neustein H, Munsat T. Tubular aggregates in subclinical alcoholic myopathy. Neurology (Minneap) 1975;25:405-12.

${ }^{17}$ Negro ADV, Angulo JM, Pomar JMR, Errasti CA. Tubular aggregates in skeletal muscle of chronic alcoholic patients. Acta Neuropathol (Berl) 1982;56:250-4.

${ }^{18}$ Dunkle LM, Diggs CH, Bergman RA, Johns RJ. A light and electron microscopic study of a second case of hypokalemic periodic paralysis with hyperthyroidism. Hopkins Med J 1970;126:225-36.

${ }^{19}$ Faugere MC, Pellissier JF, Toga M. Subsequent morphological changes in periodic paralysis: A study of seven cases. Act Neurolpathol (Berl) 1981;VII:301-4.

${ }^{20}$ Dyken M, Zeman W, Rusche T. Hypokalemic periodic paralysis, children with permanent myopathic weakness. Neurology (Minneap) 1969;19:691-9.

${ }^{21}$ Rosenberg NL, Neville HE, Ringel SP. Tubular aggregates: Their association with clinical symptomatology. Neurology (NY) 1983;33(Suppl 2):236.

${ }^{22}$ Morgan-Hughes JA, Mair WGP, Lascelles PT. A disorder of skeletal muscle associated with tubular aggregates. Brain 1970;93:873-80.

${ }^{23}$ Brumback RA, Staton RD, Susag ME. Exercise-induced pain, stiffness, and tubular aggregates in skeletal muscle. J Neurology, Neurosurg, Psychiatry 1981; 44:250-4.

${ }^{24}$ Smith R, Hughes R, Borensztajn J, et al. Focal muscle cramp in a young man, possible myopathy with tubular aggregates. Chest 1983;84:795-800.

${ }^{25}$ Lewis PD, Pallis C, Pearse AGE. "Myopathy" with tubular aggregates. $J$ Neurol Sci 1971;13:381-8.

${ }^{26}$ Lazaro RP, Fenichel GM, Kilroy AW, et al. Cramps, muscle pain, and tubular aggregates. Arch Neurol 1980;37:715-7.

${ }^{27}$ Gibbels E, Henke U, Shadlich HJ, et al. Cylindrical spirals in skeletal muscle: A further observation with clinical, morphological and biochemical analysis. Muscle Nerve 1983; 6:646-55.

${ }^{28}$ Bove KE, Iannaclone ST, Hilton PK, Samaha F. Cylindrical spirals in a familial neuromuscular disorder. Ann Neurol 1980;7:550-6.

${ }^{29}$ McDougall J, Wiles CM, Edwards RHT. Spiral membrane cylinders in the skeletal muscle of a patient with melorheososis. Neuropathol Appl Neurobiol 1980;6:69-74.

${ }^{30}$ Schiaffino S, Severin E, Cantini M, Sartore S. Tubular aggregates induced by anoxia in isolated rat skeletalo muscle. Lab Investigation 1977;37:223-8.

${ }^{31}$ Duchen LW. Changes in the electron microscopic structe ture of slow and fast skeletal muscle fibres of the mouse after the local injection of botulinum toxin. $J$ Neurol Sci 1971;14:61-74.

${ }^{32}$ Duchen LW. The local effects of tetenus toxin on the electron microscopic structure of skeletal muscle fibres of the mouse. J Neurol Sci 1973;19:169-77.

${ }^{33}$ Fardeau M, Tome FMS, Simon P. Muscle and nerve changes induced by perhexiline maleate in man and mice. Muscle Nerve 1979;2:24-36.

${ }^{34}$ Craig ID, Allen IV. Tubular aggregates in murine dystrophy heterozygotes. Muscle Nerve 1980;3:134-40.

${ }^{35}$ Cullen MJ, Weightman D. The ultrastructure of normal human muscle in relation to fibre type. J Neurol Sci 1975;25:43-56.

${ }^{36}$ Streter FA. Comparative studies on white and red muscle fractions. Fed Proc 1964;23:930-2.

${ }^{37}$ Price HM, Howes EL, Blumberg JA. Ultrastructural alteration of skeletal muscle fibres injury by cold: The acute degenerative changes. J Lab Invest 1964; 13:1264-78.

${ }^{38}$ MacDonald RD, Rencastle NB, Humphrey JG. The myopathy of hyperkalemic period paralysis. Arch Neurol 1968;19:274-84.

${ }^{39}$ Mills KR, Edwards RHT. Investigation strategies for muscle pain. J Neurol Sci 1983;58:73-88. 